Revista Interdisciplinaria de Humanidades, Educación, Ciencia y Tecnología

Año VII. Vol. VII. N²2. Edición Especial II. 2021

Hecho el depósito de ley: pp201602FA4721

ISSN-L: 2542-3029; ISSN: 2610-802X

Universidad Nacional Experimental Francisco de Miranda (UNEFM). Santa Ana de Coro. Venezuela

Gabriela Verónica Sevilla-Galarza; Lenyn Geovanny Vásconez-Acuña

\title{
Impacto del COVID-19 en la economía de los productores del sector de textiles y confecciones
}

\section{Impact of COVID-19 on the economy of textile and clothing producers}

\author{
Gabriela Verónica Sevilla-Galarza \\ gabriela.sevilla.00@est.ucacue.edu.ec \\ Universidad Católica de Cuenca. Cuenca \\ Ecuador \\ https://orcid.org/0000-0001-5688-8764 \\ Lenyn Geovanny Vásconez-Acuña \\ lenyn.vasconez@ucacue.edu.ec \\ Universidad Católica de Cuenca, Cuenca \\ Ecuador \\ https://orcid.org/0000-0001-9258-3255
}

Recibido: 20 de mayo de 2021

Aprobado: 25 de agosto de 2021 


\title{
RESUMEN
}

El Covid-19 ha producido un fuerte impacto en la economía de las empresas; y es de vital importancia el análisis de los efectos de la crisis en los estados financieros de las organizaciones mediante el uso de herramientas económicas para evaluar el nivel de liquidez, rentabilidad, endeudamiento y solvencia que poseen los productores de los distintos sectores, de manera particular del sector textiles y confecciones de la ciudad de Pelileo-Ecuador, a partir de estos resultados se bosquejó un plan financiero para atenuar el impacto de la crisis. Metodológicamente de alcance descriptivo, se aplicaron encuestas y entrevistas a las personas que conforman la unidad de análisis constituida por asociaciones de textileros registradas en la Superintendencia de Economía Popular y Solidaria; los resultados evidencian que la pandemia afectó al flujo de efectivo, redujo las ventas, elevó la cartera vencida y el endeudamiento.

Descriptores: Administración financiera; recursos financieros; recursos económicos. (Palabras tomadas de Tesauro Unesco).

\begin{abstract}
Covid-19 has had a strong impact on the economy of companies; and it is vitally important to analyze the effects of the crisis on the financial statements of organizations through the use of economic tools to assess the level of liquidity, profitability, indebtedness and solvency that producers in different sectors have, particularly of the textile and clothing sector of the city of Pelileo-Ecuador, based on these results a financial plan was drafted to mitigate the impact of the crisis. Methodologically descriptive in scope, surveys and interviews were applied to the people who make up the unit of analysis made up of textile associations registered in the Superintendency of Popular and Solidarity Economy; The results show that the pandemic affected cash flow, reduced sales, increased the overdue portfolio and increased debt.
\end{abstract}

Descriptors: Financial administration; financial resources; economic resources. (Words taken from Unesco Thesaurus). 


\section{INTRODUCCIÓN}

En el mes de diciembre de 2019, en Wuhan ciudad de China apareció el virus denominado SARS-COV-2 que en solo tres meses puso de cabeza al mundo con miles de personas contagiadas, hospitales abarrotados de pacientes con problemas respiratorios, numerosas muertes, supermercados desabastecidos, despidos intempestivos, quiebre de empresas y economías colapsadas. El director general de la Organización Mundial de la Salud (OMS) declaró que él Coronavirus Covid-19 pasa a ser una pandemia a nivel mundial, entre los países más afectados se encuentran China, Estados Unidos, Brasil y con mayor intensidad los del viejo continente (Fernández, 2020) Los gobiernos a consecuencia de la oleada de contagios tuvieron la necesidad de incrementar medidas de prevención, tales como: el distanciamiento, aislamiento, paralización de actividades, cierre de fronteras, suspensión de eventos sociales para evitar que el virus siga afectando a la salud de sus habitantes; como resultado de las disposiciones adoptadas por los mandatarios, acarreó repercusiones económicas en las pequeñas y medianas empresas (Pymes) (Huilcapi-Masacón, Troya-Terranova, \& Ocampo-Ulloa, 2020).

Las Pymes en especial las que se dedican a la producción textil han sufrido un golpe fuerte en su economía debido a la suspensión de los pedidos, paralización parcial o definitiva de la producción, decremento de las ventas, falta de rotación de inventarios, escasez de materia prima, aumento de gastos, despidos de empleados, riesgo de contagio del personal, renuncias del personal operativo ocasionan una disminución notable en los ingresos y ganancias, cierre de empresas (Organización Internacional del Trabajo [International Labour Organization], 2020).

Ecuador no se encuentra exento de la crisis sanitaria a consecuencia de la pandemia, los sistemas de salud colapsaron por el aumento indiscriminado de pacientes graves; además, de los problemas económicos y sociales que se venían arrastrando desde el año 2019, por las medidas económicas implantadas por el Gobierno Nacional, reflejado en un déficit fiscal y estancamiento económico de un $0.05 \%$ de PIB nacional con relación 
al año 2018. La declaración de estado de excepción del gobierno ecuatoriano para mitigar el número de contagios y muertes a consecuencia de la crisis sanitaria agravo las condiciones de vida de los ecuatorianos; el Ministerio de Producción, Comercio Exterior, Inversiones y Pesca, indicó que las siete primeras semanas de confinamiento en el sector económico redujo las ventas en un promedio del 42\% que equivalente 1,505 millones de dólares de pérdida. En consecuencia, los sectores más afectados fueron el comercio, manufactura y servicios con una disminución en sus ventas en más de 200 millones de dólares. Las medidas de aislamiento y distanciamiento provocaron que disminuya el comercio, las empresas cerraran y por consiguiente las personas no pudieron trabajar afectando los ingresos de las familias ecuatorianas limitando el poder adquisitivo (Red de Instituciones Financieras de Desarrollo [Network of Financial Development Institutions], 2020).

Acorde a lo antes mencionado, el problema que se plantea en la presente investigación surge de la interrogante: ¿cómo incidió el COVID-19 en los estados financieros de los productores del sector de textiles y confecciones, en la ciudad de Pelileo, en el año 2020?; $y$, como objetivo general, se propone analizar el impacto del Covid-19 en los estados financieros de los productores del sector de textiles y confecciones para la determinación de la situación económica en el año 2020.

\section{Referencial Teórico}

\section{El análisis financiero como estrategia empresarial en tiempos de crisis y salubridad} El análisis financiero es una herramienta efectiva para evaluar el desempeño económico de una empresa en un determinado período de tiempo, es decir, evalúa la posición en la que se encuentra en el presente, en el pasado y proyecta los resultados en el futuro (Nava-Rosillón, 2009). Su importancia radica en la aplicación de ciertos cálculos para determinar los aspectos fuertes y débiles al interior de una organización y las oportunidades y amenazas del entorno. Además en el entorno actual se debe tomar en 
Gabriela Verónica Sevilla-Galarza; Lenyn Geovanny Vásconez-Acuña

consideración las nuevas tendencias estratégicas y económicas para alcanzar la solidez económica a largo plazo mediante la toma de decisiones (Arrarte-Mera, 2004).

En estos tiempos difíciles de pandemia es transcendental que se efectúe un análisis de los balances financieros (estado de situación financiera, estado de resultados y estado de flujo de caja) como estrategia empresarial mediante un compendio de técnicas y herramientas analíticas para generar tácticas relevantes para la toma de decisiones, tales como: indicadores financieros (liquidez, solvencia, rotación de activos e inventarios, rotación de cuentas por cobrar, capacidad de endeudamiento, rentabilidad), sistema de Dupont y el estado de flujo de efectivo; para diagnosticar la condición financiera de las pequeñas y medianas empresas; los autores (Mosquera-Choco, 2019) y (GonzálezLemus, 2020) concuerdan que es crucial estudiar el presente y comparar el desempeño de ciclos pasados mediante la aplicación de distintas técnicas (ver figura 1), para tomar las medidas adecuadas y así mejorar los resultados en el futuro. 
Las técnicas de análisis financiero más relevante se presentan en la figura 1.

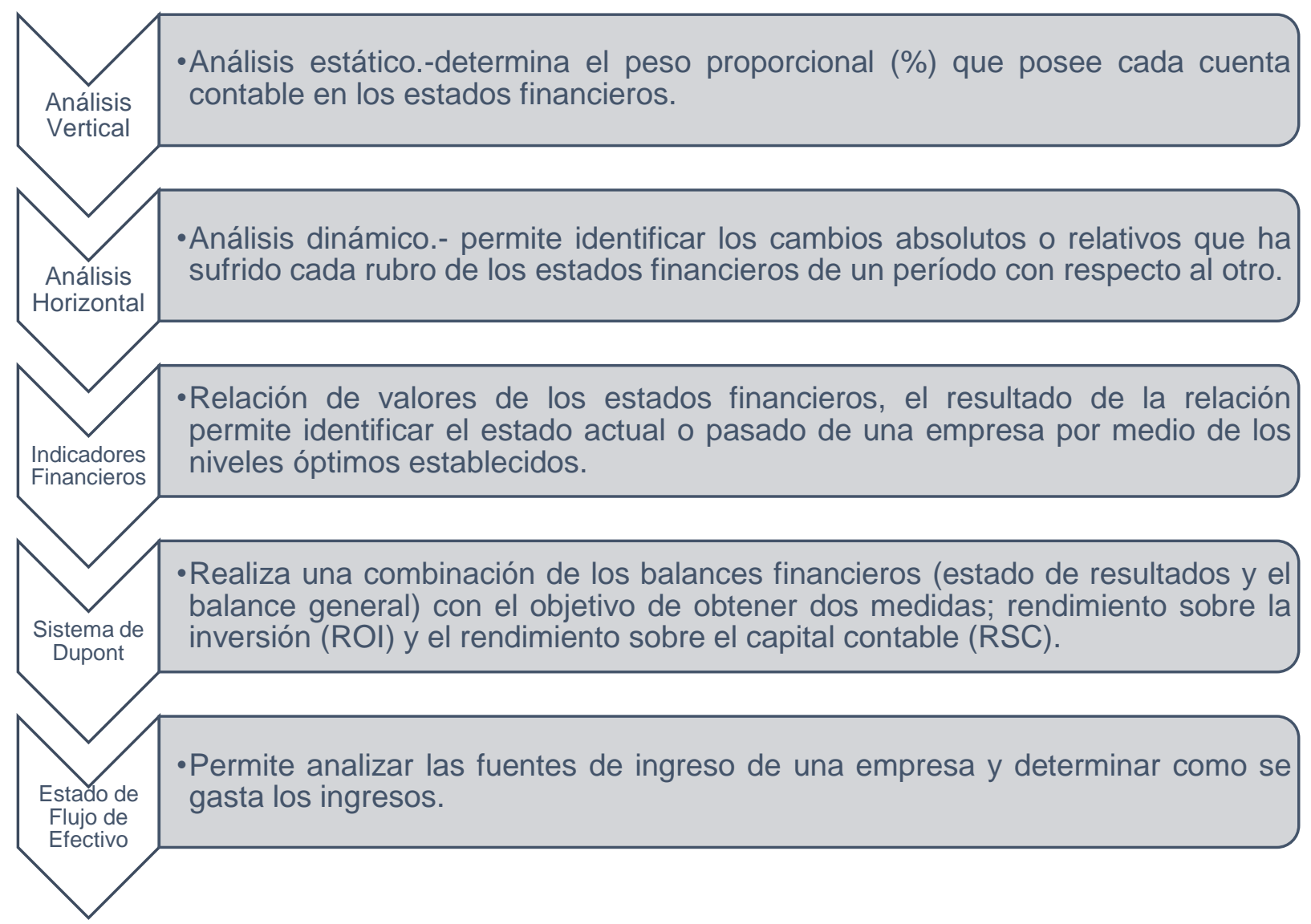

Figura 1. Técnicas de Análisis Financiero.

Fuente: Adaptado de Nava-Rosillón (2009); Ricra-Milla (2014); González-Lemus (2020).

Método horizontal y vertical: el análisis horizontal o dinámico se aplica a la información de los estados financieros correspondiente a un número de años o períodos consecutivos. Se puede utilizar este tipo de análisis para comparar los datos del año actual con los datos de otro año base, resaltando del análisis los aumentos y disminuciones; este análisis se realiza utilizando los balances financieros comparables, incluyendo la comparación de cifras de un mismo rubro de un período a otro; y, 
determinando la magnitud de aumento y disminución para realizar proyecciones futuras. Análisis vertical o estático: consiste en tomar un estado financiero y relacionar el total de cuentas con el subgrupo de cuentas o del total de una partida; el objetivo es determinar porcentualmente la participación de cada cuenta con respecto al total y observar su comportamiento a lo largo de la vida de la empresa (Ballesteros-Cerchiaro, 2017). Indices financieros: los indices financieros fundamentales para la determinación del estado económico de un negocio se clasifican en; liquidez, solvencia, endeudamiento y rentabilidad.

Liquidez: dentro de los varios impactos que ha generado la pandemia a las empresas, está la falta de liquidez a causa del exceso de deudas y escasos ingresos; además, otros factores que inciden son la acumulación de cuentas por cobrar, el otorgamiento de nuevos plazos de pago a los clientes, el incremento de costos y ganancias muy reducidas (Espinoza-Carrión, 2019), lo cual repercute en la capacidad de pago; y por consiguiente, la organización no puede cubrir con sus deudas a corto plazo en un determinado período de tiempo (Jaramillo, 2021). Los autores (González-Lemus, 2020; Mejía-Hidalgo, 2020) coinciden que es óptimo medir la liquidez, ya que permite evaluar la capacidad que posee la empresa para enfrentar sus obligaciones a corto y largo plazo.

Solvencia: determinar la solvencia que posee una empresa es indispensable, porque se puede medir la capacidad de endeudamiento o el nivel de recursos que se encuentran comprometidos. Los problemas que tienen los empresarios como efecto de la pandemia por COVID-19 recaen en las dificultades para encontrar financiación, además de la lentitud en la asignación de créditos por parte de las instituciones financieras (Touza \& Picatoste, 2020).

Rentabilidad: el análisis de rentabilidad es clave a la hora de evaluar y ejecutar proyectos de inversión, puesto que, permite examinar la capacidad de generar beneficios y los retornos de una inversión, ya sea este de renta fija o variable, además se emplea como métrica para deducir el retorno de inversiones no financieras; y, el uso eficaz de los recursos (Barreno, 2013). 
Endeudamiento: el endeudamiento en las empresas es un arma de doble filo, ya que, permite invertir o cubrir los gastos o costos. En medio de la crisis sanitaria causada por el distanciamiento social, al tomar créditos se logró limitar los despidos; el endeudamiento aumento aún más como consecuencia de las políticas de respaldo para mitigar la pandemia (Fondo Monetario Internacional, 2021). Es importante este cálculo, ya que con la disponibilidad de bases de datos cuantitativos que revelan los estados financieros se puede determinar la estabilidad, sostenibilidad y el nivel del riesgo para cubrir las obligaciones del negocio a medio y largo plazo y asegurar que la empresa sea solvente (Master MBA Sevilla, 2020).

Sistema DuPont: es un diagrama de fórmulas que facilita la visualización de la descomposición de los rendimientos de activos y de capital en diferentes etapas; muestra la relación entre estos elementos y las variables económicas que los afectan; admite clasificar y descomponer cada factor de una manera más digerible para desarrollar los objetivos, maximizar ganancias de la empresa e identificar en donde necesita mejorar sus operaciones con el fin de elevar la rentabilidad (García-Jiménez, 2014).

Estado de flujo de efectivo: ha traído algunos problemas la crisis económica que se enfrenta en la actualidad, tales como fuerte caída de la demanda, ausencia de ventas, alto nivel de endeudamiento, entre otros. En este contexto, para dotar a los empresarios de información respecto de los recursos que posee la empresa se debe elaborar el estado de flujo de efectivo, el cual permite conocer la capacidad para cumplir las necesidades de financiación y las obligaciones contraídas; determinar el impacto económico en las acciones de inversión y financiamiento; identificar falta o exceso de liquidez; evaluar las actividades que la empresa invierte dinero, ante lo expuesto (Guzmán-Maldonado, 2020) y (Apuntes Empresariales ESAN, 2020) consideran que el estado de flujo es una herramienta de análisis financiero efectiva ante la crisis.

Ante el escenario expuesto, la realidad empresarial para los productores de textiles y de confecciones se ha visto afectada por la pandemia, y es indispensable que se realice una valoración de la información plasmada en los balances de información financiera 
mediante un análisis económico y, el cálculo y análisis de herramientas financieras para descubrir los efectos producidos como estrategia para la toma de decisiones, en este sentido, es de vital importancia aplicar un plan financiero, el cual permita garantizar los niveles de liquidez, rentabilidad, solvencia, endeudamiento y flujo de efectivo necesarios para mantener las operaciones en marcha.

\section{Cálculo y análisis de los indicadores financieros en tiempos de pandemia COVID-} 19

El análisis financiero se fundamenta en el cálculo de ratios financieros que muestran la posición de liquidez, solvencia, rotación de inventarios, eficacia, endeudamiento, rentabilidad, flujos de efectivo para la interpretación de la información, obtenida para la determinación de decisiones oportunas, en la actual coyuntura (González-Lemus, 2020). El indicador de liquidez se utiliza para establecer la capacidad que posee una organización para afrontar los compromisos contraídos a corto plazo, es decir, cuanto más elevado es el indicador, aumenta la posibilidad de que la empresa consiga derogar las deudas a corto plazo (Padilla-Martínez, Quispe-Otacama, \& Telencha-Santos, 2017); en la tabla 1 se presenta la fórmula de cálculo y su interpretación.

\section{Tabla 1.}

Fórmula de los ratio de liquidez.

\begin{tabular}{cclc}
\hline Ratio & Fórmula & \multicolumn{1}{c}{ Interpretación } & Valores óptimos \\
\hline Liquidez corriente $=$ & $\begin{array}{l}\text { Si el resultado es superior a 1 la } \\
\text { empresa posee una adecuada }\end{array}$ & \\
\cline { 2 - 5 } & Activo Corriente & $\begin{array}{l}\text { capacidad para hacer frente a } \\
\text { sus obligaciones a corto plazo; } \mathrm{Si}\end{array}$ & Entre 1.4-1.8 \\
& $\begin{array}{l}\text { el resultado es menor a 1 la } \\
\text { empresa no puede cubrir con sus } \\
\text { obligaciones a corto plazo. }\end{array}$ & \\
\hline
\end{tabular}

Fuente: Adaptado de Ricra-Milla (2014); Navarro (2019). 
Solvencia. - pondera la capacidad de la organización para generar dinero y cubrir sus obligaciones, la fórmula e interpretación se presenta en la tabla 2:

\section{Tabla 2.}

Fórmula de ratios de solvencia.

\begin{tabular}{cccc}
\hline Ratio & \multicolumn{1}{c}{ Fórmula } & \multicolumn{1}{c}{ Interpretación } & Valores óptimos \\
\hline Solvencia $=$ & Activo Total & $\begin{array}{l}\text { Si el resultado es 1, quiere } \\
\text { decir que la empresa cuenta } \\
\text { con 1 dólar en su activo, por } \\
\text { cada dólar que adeuda. }\end{array}$ & Mayor a 1,5 \\
\cline { 2 - 4 } & & & \\
\hline
\end{tabular}

Fuente: Adaptado de Ballesteros-Cerchiaro, (2017); González (2021).

Rotación de activos e inventarios. - indica el número de veces que se adquiere y se vende mercadería y el número de días que se tarda el proceso, ver fórmula e interpretación en la tabla 3:

\section{Tabla 3.}

Fórmula de rotación e inventarios.

\begin{tabular}{|c|c|c|c|}
\hline Ratio & Fórmula & Interpretación & Valores óptimos \\
\hline Rotación de inventarios= & $\frac{\text { Costo de Ventas }}{\text { Promedio de Inventarios }}$ & $\begin{array}{l}\text { Si el resultado es mayor a } 1 \text { la } \\
\text { empresa rota su inventario por } \\
\text { lo menos una vez al año; y si } \\
\text { el resultado es menor a } 1 \text { la } \\
\text { empresa no logró rotar sus } \\
\text { inventarios. }\end{array}$ & Mayor a 1 \\
\hline Número de días = & $\frac{\text { Días del período }}{\text { Rotación de Inventarios }}$ & & \\
\hline
\end{tabular}

Fuente: Adaptado de: Coronel-Morocho (2019); González (2021). 
Rotación de las cuentas por cobrar. - indica el número de veces que se facilita a los clientes financiación y lo que se demoran en cancelar lo adeudado, ver fórmula e interpretación de la tabla 4:

\section{Tabla 4.}

Fórmula de rotación de cuentas por cobrar

\begin{tabular}{rlll}
\hline \multicolumn{1}{c}{ Ratio } & \multicolumn{1}{c}{ Fórmula } & \multicolumn{1}{c}{ Interpretación } & Valores óptimos \\
\hline Rotación de cuentas & $\begin{array}{l}\text { Ventas realizadas } \\
\text { prédito }\end{array}$ & $\begin{array}{l}\text { Es la medida de la celeridad y } \\
\text { el poder que una empresa } \\
\text { tiene para cobrar a sus } \\
\text { clientes las ventas a crédito. }\end{array}$ & Mayor a 1 \\
Número de días $=$ & $\begin{array}{l}\text { Promedio de cuentas } \\
\text { por cobrar }\end{array}$ & $\begin{array}{l}\text { Días de período } \\
\text { Rotación de cuentas } \\
\text { por pagar }\end{array}$ & \\
\hline
\end{tabular}

Fuente: Adaptado de Robles-Román, (2012); Ricra-Milla (2014).

Rentabilidad. - indica la capacidad que posee una empresa para generar utilidades, ver fórmula e interpretación de la tabla 5 :

\section{Tabla 5.}

Fórmula de rentabilidad.

\begin{tabular}{clllc}
\hline Ratio & \multicolumn{1}{c}{ Fórmula } & \multicolumn{1}{c}{ Interpretación } & Valores óptimos \\
\hline Rentabilidad de activos $=$ & Renta Neta $\times 100$ & $\begin{array}{l}\text { Si el coeficiente es alto la } \\
\text { empresa posee mayor } \\
\text { rentabilidad }\end{array}$ & Mayor a 1 \\
\cline { 2 - 5 } & Activos Totales & & \\
\hline
\end{tabular}

Fuente: Adaptado de Robles-Román (2012) ; Ricra-Milla (2014). 
Endeudamiento. - mide la simetría en que una organización está sujeta a terceros, ver fórmula e interpretación de la tabla 6 :

\section{Tabla 6.}

Fórmula de endeudamiento.

\begin{tabular}{|c|c|c|c|}
\hline Ratio & Fórmula & Interpretación & Valores óptimos \\
\hline \multirow[t]{2}{*}{ Endeudamiento = } & $\frac{\text { Total Pasivo }}{\text { Total Activo }}$ & $\begin{array}{l}\text { Por cada } 1,00 \text { USD que la } \\
\text { organización tiene invertido } \\
\text { en activos, porcentaje (\%) es }\end{array}$ & \\
\hline & & $\begin{array}{lll}\text { financiado } & \text { por los } \\
\text { acreedores. } & & \end{array}$ & Entre 0,4 y 0,6 \\
\hline
\end{tabular}

Fuente: Adaptado de Lozano-Meade (2020).

Sistema de DuPont.- es el análisis de la ocupación económica y operativa de una empresa, mediante la relación de algunas razones financieras tanto de actividad como de rotación de activos totales, apalancamiento financiero y el margen de utilidad sobre las ventas, para determinar la eficiencia de la utilización de los activos y capital de trabajo (Alcázar-Ponce, 2015).

Los elementos del sistema de Dupont son dos: el Return on Equity (ROE) y el Return on Assets (ROA) ROE o rentabilidad financiera es un indicador que valora el rendimiento del capital, es decir, valora la utilidad que genera la empresa sobre sus fondos propios. ROA o rentabilidad sobre los activos es un indicador que mide la utilidad del total de los activos de la empresa, ver tabla 7 : 


\section{Tabla 7.}

Fórmula del sistema de DuPont.

\begin{tabular}{lll}
\hline Nombre & \multicolumn{1}{c}{ Fórmula } \\
\hline ROE $=$ & $($ renta neta/ventas $) *($ ventas/activo total $) *($ activo total/ patrimonio $)$ \\
& ROA $=$ & $($ utilidad neta/activos total $)$ \\
\hline
\end{tabular}

Fuente: Adaptado de Alcázar-Ponce (2015).

Flujo de efectivo.- es la variación de todo el efectivo que llega a un negocio, ya sea, por cobros, inversiones, pagos de dividendos, en otros términos, es todo el dinero que tiene tesorería por sus actividades operacionales regulares y, la salida de dinero en un determinado período de tiempo (Moncayo, 2016). Existe dos métodos de cálculo el directo e indirecto.

El método directo consiste en volver a realizar el estado de resultados basándose en el sistema de caja para determinar las actividades de operación incluyendo las transacciones realizadas en efectivo (Asociación para el progreso de la dirección (APD), 2019), ver tabla 8:

\section{Tabla 8.}

Método directo del flujo de efectivo

Nombre Fórmula

Flujo de efectivo $=$ Ingresos - pagos - gastos - interés - impuestos

Fuente: Adaptada de Asociación para el progreso de la dirección (APD) (2019). 
El método indirecto (ver tabla 9) consiste en partir de los ingresos netos y realizar cambios en función del estado de situación financiera (Asociación para el progreso de la dirección (APD), 2019):

\section{Tabla 9.}

Método indirecto del flujo de efectivo.

\begin{tabular}{cl}
\hline Nombre & \multicolumn{1}{c}{ Fórmula } \\
\hline Flujo de efectivo $=$ & $\begin{array}{l}\text { Ingreso neto }+ \text { pérdidas y renta por inversiones }- \text { financiación }+ \text { cargos no } \\
\text { monetarios }+ \text { cambio en las cuentas de operación }\end{array}$ \\
\hline
\end{tabular}

Fuente: Adaptada de Asociación para el progreso de la dirección (APD), (2019).

El análisis económico - financiero permite observar un panorama más amplio de la situación económica de las empresas, contexto que se ve reflejado en los balances económicos mediante el cálculo de herramientas financieras para conocer el estado de la liquidez, solvencia, eficiencia, utilidades y el flujo de caja de la empresa; especialmente en la crisis de salubridad actual, la importancia de realizar estos cálculos radica en que se puede cuantificar el impacto que generaron las medidas sanitarias tomadas por los gobiernos en los balances económicos de los productores de textiles y confecciones.

\section{Impacto del Covid-19 en los estados financieros}

El gobierno ecuatoriano para frenar los contagios masivos y decesos de los habitantes debido al virus, implementó medidas sanitarias tales como: el distanciamiento, aislamiento y paralización de actividades para frenar los efectos en la población; situación que se vio reflejada en problemas empresariales relacionados con la liquidez, rentabilidad, solvencia, flujos de caja; entre otros. La activación parcial de actividades económicas trae incertidumbre a los empresarios sobre si los costos para la implantación 
de las medidas de seguridad aumentaran y si pueden cubrirlos con los ingresos que poseen o si el negocio puede seguir en marcha. Esta situación se refleja en la contabilidad y en los resultados de los estados financieros.

Las firmas privadas (Deloitte, 2020; Ernst \& Young EY, 2020; PricewaterhouseCoopers PWC, 2020) concuerdan que las implicaciones en los reportes contables y en las Normas Internacionales de Información Financiera (NIIF) o Normas Internacionales de Contabilidad (NIC) son las expuestas en la tabla 10.

Tabla 10.

Implicaciones en los reportes contables por la pandemia de COVID-19.

\begin{tabular}{|c|c|c|}
\hline Tema & NIIF o NIC & Impacto \\
\hline $\begin{array}{l}\text { Activos no } \\
\text { financieros }\end{array}$ & NIC 1 & $\begin{array}{l}\text { Se considera deterioro cuando una empresa no logra recuperar el } \\
\text { valor que se encuentra registrado en libros mediante el uso o } \\
\text { enajenación; la medición se lo realiza mediante el cálculo del valor } \\
\text { razonable menos los costos de enajenación; y, la estimación } \\
\text { cuando el activo se encuentra en uso se calcula mediante la } \\
\text { estimación de las entradas y salidas originadas del uso y el } \\
\text { descuento. }\end{array}$ \\
\hline $\begin{array}{l}\text { Activos } \\
\text { contingentes }\end{array}$ & NIC 37 & $\begin{array}{l}\text { Como medida de prevención por el contagio algunas empresas } \\
\text { cerraron y suspendieron las actividades, por esta razón los } \\
\text { negocios al contratar un seguro por contingentes pueden recuperar } \\
\text { las pérdidas que se generaron por el cierre de las actividades. }\end{array}$ \\
\hline Arrendamientos & NIIF 16 & $\begin{array}{l}\text { Tanto el arrendador como el arrendatario podrá negociar y realizar } \\
\text { concesiones, ampliaciones de plazo, costo. En los contratos de } \\
\text { arrendamiento es menester determinar el tratamiento adecuado de } \\
\text { las modificaciones realizadas a los contratos en la contabilización } \\
\text { de las condonaciones o aplazamientos de los pagos de } \\
\text { arrendamiento. }\end{array}$ \\
\hline Inventarios & NIC 2 & $\begin{array}{l}\text { Los inventarios requieren que se reduzca al valor neto realizable, } \\
\text { es decir, al menor precio debido a la reducción de las ventas } \\
\text { esperadas. Además, se requiere que los gastos de producción que } \\
\text { son fijos se añadan en el costo de inventarios de acuerdo a la } \\
\text { producción normal. }\end{array}$ \\
\hline $\begin{array}{l}\text { Propiedad, planta y } \\
\text { equipo }\end{array}$ & NIC 16 & $\begin{array}{l}\text { Hay que realizar un estudio de la propiedad, planta y equipo sobre } \\
\text { la subutilización y utilización en un determinado período de tiempo. } \\
\text { Es necesario que la depreciación se considere en el estado de } \\
\text { resultados entre tanto el activo se encuentra inactivo. }\end{array}$ \\
\hline $\begin{array}{l}\text { Subvenciones del } \\
\text { gobierno }\end{array}$ & NIC 20 & $\begin{array}{l}\text { Son ayudas gubernamentales concedidas a las empresas como } \\
\text { subsidios, exenciones, ampliaciones de plazo, reducciones en }\end{array}$ \\
\hline
\end{tabular}




\begin{tabular}{|c|c|c|}
\hline $\begin{array}{l}\text { Amortización de } \\
\text { pérdidas fiscales }\end{array}$ & NIC 12 & $\begin{array}{l}\text { Las empresas deben tomar en cuenta las pérdidas generadas por } \\
\text { las medidas tomadas para evitar los contagios, el deterioro } \\
\text { económico y considerar su revelación en un hecho posterior. }\end{array}$ \\
\hline Contratos onerosos & NIC 37 & $\begin{array}{l}\text { Se considera contratos onerosos a los costos que no se puede } \\
\text { evitar producto de las obligaciones que exceden los beneficios } \\
\text { económicos. Los contratos a ejecutarse se deben revisar para } \\
\text { determinar si existen condiciones. }\end{array}$ \\
\hline $\begin{array}{l}\text { Valoración de } \\
\text { Inventarios }\end{array}$ & NIC 2 & $\begin{array}{l}\text { Los inventarios requieren que se realice una valoración al menor } \\
\text { costo o valor neto. }\end{array}$ \\
\hline $\begin{array}{l}\text { Reconocimientos } \\
\text { de ingresos }\end{array}$ & NIIF 15 & $\begin{array}{l}\text { Las ventas sufrieron una reducción, hay que considerar que la } \\
\text { demanda ha disminuido y existen devoluciones de productos y } \\
\text { cancelación de servicios, así como concesiones en los precios de } \\
\text { venta, multas. Se registrarán los ingresos en la contabilidad, } \\
\text { cuando el cliente cancele. }\end{array}$ \\
\hline $\begin{array}{l}\text { Revelación de } \\
\text { hechos } \\
\text { subsecuentes }\end{array}$ & NIC 10 & $\begin{array}{l}\text { Afecta a los balances financieros en el reconocimiento y medición } \\
\text { de los activos y pasivos si estos ocurrieron posterior a la } \\
\text { presentación de los informes. }\end{array}$ \\
\hline Negocio en marcha & NIC 1 & $\begin{array}{l}\text { Es la certeza de que un negocio tiene la capacidad de seguir en } \\
\text { marcha, situación que debe ser revelada en los estados } \\
\text { financieros. }\end{array}$ \\
\hline Cartera de crédito & NIC 39 & $\begin{array}{l}\text { Hay que considerar la situación económica de los acreditados e } \\
\text { incorporar condiciones de aplazamiento de pagos y mora. } \\
\text { Incorporar ajustes de garantías que sean dinámicos y que afecten } \\
\text { a las estimaciones. }\end{array}$ \\
\hline Cuentas por cobrar & NIIF 9 & $\begin{array}{l}\text { Se debe estimar la pérdida por remanente del activo por las } \\
\text { condiciones actuales; y, aumentar estimaciones de deterioro como } \\
\text { efecto de la operación. }\end{array}$ \\
\hline $\begin{array}{l}\text { Beneficios para } \\
\text { empleados }\end{array}$ & NIC 37 & $\begin{array}{l}\text { Se requiere se realice un plan y se determine el valor presente de } \\
\text { las obligaciones de los trabajadores para tener un valor razonable } \\
\text { y evitar sobrestimaciones. }\end{array}$ \\
\hline $\begin{array}{l}\text { Costeo de } \\
\text { inventarios }\end{array}$ & NIC 2 & $\begin{array}{l}\text { Es menester que se realice la valoración de los costos de acuerdo } \\
\text { con la capacidad de producción. }\end{array}$ \\
\hline
\end{tabular}

impuestos y créditos a interés bajo. Se reconocen en el balance general cuando las subvenciones son activos y cuando son utilidades en el estado de resultados. 
Las paralizaciones parciales o totales de las operaciones como efecto de la pandemia afectan a los negocios en la generación de ingresos, en la recuperación de las cuentas por cobrar, en el deterioro de inventarios y activos fijos, aumentando los costos de paralización y los gastos de reactivación; acarrea efectos considerables en las cuentas de los balances económicos; por lo que, es preciso medir el impacto mediante un análisis económico financiero para tener una visión de lo que está sucediendo con la empresa y así tomar decisiones acertadas y establecer estrategias financieras sólidas para que los negocios continúen en marcha.

\section{METODOLOGÍA}

El alcance fue descriptivo, en vista que, permitió describir los efectos en la economía de los productores del sector de textiles y confecciones en la ciudad de Pelileo, de corte transversal, debido a que, el levantamiento de datos se realizó en un período de tiempo. El universo estuvo conformado por 144 personas; 126 directivos y 18 contadores de las asociaciones de textileros registradas en la Superintendencia de Economía Popular y Solidaria de la ciudad de Pelileo. Las técnicas utilizadas para la recopilación de la información fueron la encuesta y la entrevista; de los cuales, los instrumentos fueron el cuestionario y la guía de preguntas. Se aplicó estadística descriptiva para el análisis de los datos.

\section{RESULTADOS}

Los efectos económicos provocados por el Covid-19 en las empresas son diversos a nivel mundial, a consecuencia de que las medidas adoptadas por los gobernantes en especial por las paralizaciones de actividades, lo cual ha repercutido en la liquidez, rentabilidad y solvencia de las organizaciones en el Ecuador, en este sentido, es pertinente valorar el impacto económico de la pandemia en las asociaciones de productores textiles y confecciones en la ciudad de Pelileo. Con este objetivo, se ha recopilado las respuestas 
de los principales resultados de las encuestas y entrevistas aplicadas a los contadores y presidentes de las asociaciones; y, se expone su percepción ante la crisis.

Impacto económico: el $93 \%$ de los encuestados concuerda que las medidas implantadas por el gobierno ecuatoriano (confinamiento y las declaratorias de emergencia para mitigar los contagios del Covid-19), paralizaron las actividades económicas en unos casos de manera total y otros de manera parcial, afectando así en sus finanzas.

En consecuencia, al consultarles si sus ventas se vieron comprometidas durante el año 2020 , el $84 \%$ de encuestados contestó que las ventas si disminuyeron.

Liquidez: El 100\% de los productores textileros del cantón Pelileo afirma que tienen problemas en su liquidez como consecuencia de la pandemia y, por consiguiente, en el flujo de efectivo. Además, el $100 \%$ de aseguran tener dificultades para cubrir sus obligaciones de corto plazo.

Rentabilidad: el $91 \%$ de los encuestados coincide en que tuvieron que incurrir en gastos operativos para la implementación de programas de bioseguridad para proteger la salud de sus colaboradores. Además, el $78 \%$ de productores aseguran que, como resultado del aumento de los gastos y disminución de las ventas, su rentabilidad decreció de manera notable en año 2020.

Cartera vencida: el $84 \%$ de encuestados afirma que el nivel de cartera vencida se incrementó al igual que el riesgo financiero de sus empresas.

Estrategias financieras: para mantener sus negocios en marcha durante la crisis económica, varias empresas implementaron estrategias financieras con el objetivo de mejorar sus indicadores de liquidez. Al respecto, el $61 \%$ de los investigados manifestó que a causa de la pandemia modificó sus políticas de cobranza, con la finalidad de recaudar las deudas que presenta mayor vencimiento. Además, el $52 \%$ de los empresarios aseguran que han aplicado estrategias para reducir los costos y gastos. 


\section{PROPUESTA}

En este apartado se analiza el impacto del Covid-19 en los estados financieros de los productores del sector de textiles y confecciones, considerando los siguientes pasos:

$\checkmark$ Identificación y evaluación de riesgos

$\checkmark$ Efectos contables y financieros

$\checkmark$ Estrategias financieras para superar la crisis

\section{Identificación y evaluación de riesgos}

Se realizó la identificación y la evaluación de riesgos con base en las encuestas realizadas a los productores de textiles y confecciones en la ciudad de Pelileo (ver tabla 11).

\section{Tabla 11}

Matriz de identificación y evaluación de riesgos

\begin{tabular}{|c|c|c|c|c|}
\hline Componente & $\begin{array}{l}\text { Debilidad } \\
\text { detectada }\end{array}$ & Efecto del riesgo & $\begin{array}{c}\text { Tipo } \\
\text { del } \\
\text { riesgo }\end{array}$ & $\begin{array}{l}\text { Nivel del } \\
\text { impacto }\end{array}$ \\
\hline $\begin{array}{l}\text { Resultado del } \\
\text { ejercicio }\end{array}$ & $\begin{array}{l}\text { Disminución de } \\
\text { la demanda }\end{array}$ & $\begin{array}{l}\text { Pérdida del Ejercicio. } \\
\text { Cierre de puntos de } \\
\text { venta. } \\
\text { Cierre del negocio. } \\
\text { La empresa no puede }\end{array}$ & Inherente & Alto \\
\hline Ventas & $\begin{array}{l}\text { Reducción de } \\
\text { las ventas }\end{array}$ & $\begin{array}{l}\text { cubrir con sus } \\
\text { obligaciones. } \\
\text { Llegar aspectos legales } \\
\text { con los acreedores. }\end{array}$ & Inherente & Alto \\
\hline $\begin{array}{l}\text { Cuentas por } \\
\text { cobrar }\end{array}$ & $\begin{array}{l}\text { Cartera } \\
\text { vencida }\end{array}$ & $\begin{array}{l}\text { Falta de liquidez en las } \\
\text { productoras textiles y de } \\
\text { confecciones. }\end{array}$ & Control & Alto \\
\hline Capital & Falta de Capital & $\begin{array}{l}\text { No se puede invertir en } \\
\text { la producción de nuevas } \\
\text { líneas de producción } \\
\text { debido a la falta de } \\
\text { capital. }\end{array}$ & Inherente & Alto \\
\hline
\end{tabular}


Revista Interdisciplinaria de Humanidades, Educación, Ciencia y Tecnología

Año VII. Vol. VII. N². Edición Especial II. 2021

Hecho el depósito de ley: pp201602FA4721

ISSN-L: 2542-3029; ISSN: 2610-802X

Universidad Nacional Experimental Francisco de Miranda (UNEFM). Santa Ana de Coro. Venezuela

Gabriela Verónica Sevilla-Galarza; Lenyn Geovanny Vásconez-Acuña

Financiación

Dificultad para

obtener

Nivel de endeudamiento

financiamiento

muy elevado.

Menor crecimiento y Inherente

Medio productividad

\begin{tabular}{|l|l|l|}
\hline Alto & Medio & Bajo \\
\hline & & \\
\hline
\end{tabular}

Fuente: Elaboración propia a partir de las encuestas aplicadas a los productores de textiles y confecciones.

\section{Efectos contables y financieros}

Para analizar los efectos contables se tomó como base los balances generales de dos asociaciones productoras de textiles y confecciones de la ciudad de Pelileo de los años 2019 y 2020 y se realizó un análisis de horizontal para evidenciar las tendencias de las cuentas contables como efectos del Covid-19 (ver tabla 12 y 13). 
Tabla 12.

Efectos contables - Análisis Horizontal de Balances Generales. -

\begin{tabular}{|c|c|c|c|c|c|c|c|c|c|c|c|c|c|c|}
\hline \multirow[b]{2}{*}{ Cuentas Contables/ Años } & \multicolumn{7}{|c|}{ Asociación 1} & \multicolumn{7}{|c|}{ Asociación 2} \\
\hline & & 2020 & & 2019 & & $\begin{array}{l}\text { Variación } \\
\text { Absoluta }\end{array}$ & $\begin{array}{l}\text { Variación } \\
\text { Relativa }\end{array}$ & & 2020 & & 2019 & & $\begin{array}{l}\text { Variación } \\
\text { Absoluta }\end{array}$ & $\begin{array}{l}\text { Variación } \\
\text { Relativa }\end{array}$ \\
\hline \multicolumn{15}{|l|}{\begin{tabular}{|l|} 
Activos \\
Activo corriente
\end{tabular}} \\
\hline Activo corriente & $\$$ & 2.593 .605 & $\$$ & 3.194 .599 & $\$$ & -600.994 & $-18,81 \%$ & $\$$ & 2.835 .403 & $\$$ & 3.373 .441 & $\$$ & -538.038 & $-15,95 \%$ \\
\hline Efectivo y equivalentes de efectivo & $\Phi$ & 75.781 & $\$$ & 48.242 & $\Phi$ & 27.539 & $57,09 \%$ & $\$$ & 255.819 & $\$$ & 109.673 & $\Phi$ & 146.146 & $133,26 \%$ \\
\hline Cuentas por cobrar no relacionados & $\$$ & 299.816 & $\$$ & 493.562 & $\$$ & -193.746 & $-39,25 \%$ & $\$$ & 535.002 & $\$$ & 1.068 .725 & $\$$ & -533.723 & $-49,94 \%$ \\
\hline Cuentas por cobrar relacionados & $\$$ & 1.187 .151 & $\$$ & 1.253 .340 & $\$$ & -66.189 & $-5,28 \%$ & $\$$ & - & $\$$ & & $\$$ & & \\
\hline Otras cuentas por cobrar & $\$$ & 11.544 & $\$$ & 349.853 & $\$$ & -338.309 & $-96,70 \%$ & $\$$ & - & $\$$ & & $\$$ & & \\
\hline Activos por impuestos corrientes & $\$$ & 210.502 & $\$$ & 162.767 & $\$$ & 47.735 & $29,33 \%$ & $\$$ & 93.502 & $\$$ & 66.269 & $\$$ & 27.233 & $41,09 \%$ \\
\hline Inventarios & $\$$ & 808.811 & $\$$ & 886.835 & $\$$ & -78.024 & $-8,80 \%$ & $\$$ & 1.951 .080 & $\$$ & 2.128 .774 & $\$$ & -177.694 & $-8,35 \%$ \\
\hline Activo no corriente & $\$$ & 5.062 .763 & $\$$ & 4.376 .708 & $\$$ & 686.055 & $15,68 \%$ & $\$$ & 421.712 & $\$$ & 298.154 & $\$$ & 123.558 & $41,44 \%$ \\
\hline Activo fijo & $\$$ & 5.062 .763 & $\$$ & 3.691 .105 & $\$$ & 1.371 .658 & $37,16 \%$ & $\$$ & 239.278 & $\$$ & 273.560 & $\$$ & -34.282 & $-12,53 \%$ \\
\hline Otros activos no corrientes & & & $\$$ & 685.603 & $\$$ & -685.603 & $-100,00 \%$ & $\$$ & & $\$$ & & $\$$ & & \\
\hline Activos por derecho de uso & $\$$ & & $\$$ & & $\$$ & & & $\$$ & 102.644 & & & $\$$ & 102.644 & \\
\hline Impuestos diferidos & $\$$ & & $\$$ & & $\$$ & & & $\$$ & 79.790 & $\$$ & 24.594 & $\$$ & 55.196 & $224,43 \%$ \\
\hline Total activos & $\$$ & 7.656 .368 & $\$$ & 7.571 .307 & $\$$ & 85.061 & $1,12 \%$ & $\$$ & 3.257 .115 & $\$$ & 3.671 .595 & $\$$ & -414.480 & $-11,29 \%$ \\
\hline Pasivos & & & & & $\Phi$ & & & & & & & & & \\
\hline Pasivo corriente & $\$$ & 713.063 & $\$$ & 1.224 .987 & $\$$ & -511.924 & $-41,79 \%$ & $\$$ & 1.442.984 & $\$$ & 1.878 .667 & $\$$ & -435.683 & $-23,19 \%$ \\
\hline Obligaciones con instituciones financieras & $\$$ & 276.543 & $\$$ & 624.365 & $\$$ & -347.822 & $-55,71 \%$ & $\$$ & 42.877 & $\$$ & 276.018 & $\$$ & -233.141 & $-84,47 \%$ \\
\hline Cuentas y documentos por pagar & $\$$ & 258.970 & $\$$ & 251.550 & $\$$ & 7.420 & $2,95 \%$ & $\$$ & 1.149 .930 & $\$$ & 1.290 .003 & $\$$ & -140.073 & $-10,86 \%$ \\
\hline Otras cuentas por pagar & $\$$ & 57.930 & $\$$ & 20.273 & $\$$ & 37.657 & $185,75 \%$ & $\$$ & - & $\$$ & & $\$$ & & \\
\hline Cuentas por pagar relacionadas & & & $\$$ & 3.610 & $\$$ & -3.610 & $-100,00 \%$ & $\$$ & & $\$$ & & $\$$ & & \\
\hline Beneficios empleados corto plazo & $\$$ & 74.167 & $\$$ & 200.602 & $\$$ & -126.435 & $-63,03 \%$ & $\$$ & 143.986 & $\$$ & 201.870 & $\$$ & -57.884 & $-28,67 \%$ \\
\hline Otras obligaciones corrientes & $\$$ & 45.453 & $\$$ & 124.587 & $\$$ & -79.134 & $-63,52 \%$ & $\$$ & & $\$$ & - & $\$$ & & \\
\hline Arrendamientos & $\$$ & & $\$$ & & $\$$ & & & $\$$ & 21.976 & & & $\$$ & 21.976 & \\
\hline Impuestos corrientes & $\$$ & & $\$$ & & $\$$ & & & $\$$ & 84.215 & $\$$ & 110.776 & $\$$ & -26.561 & $-23,98 \%$ \\
\hline Pasivo no corriente & $\$$ & 1.454 .481 & $\$$ & 852.696 & $\$$ & 601.785 & $70,57 \%$ & $\$$ & 455.764 & $\$$ & 548.529 & $\$$ & -92.765 & $-16,91 \%$ \\
\hline Obligaciones con instituciones financieras & $\$$ & 1.133 .909 & $\$$ & 394.665 & $\$$ & 739.244 & $187,31 \%$ & $\$$ & 218.906 & $\$$ & 32.754 & \$ & 186.152 & $568,33 \%$ \\
\hline Provisiones por beneficios a empleados & $\$$ & 320.572 & $\$$ & 458.031 & $\$$ & -137.459 & $-30,01 \%$ & $\$$ & & $\$$ & & $\$$ & & \\
\hline Cuentas por pagar accionistas & $\$$ & - & $\$$ & - & $\$$ & - & & & & $\$$ & 291.973 & $\$$ & -291.973 & $-100,00 \%$ \\
\hline Beneficios socie & $\$$ & - & $\$$ & - & $\$$ & - & & $\$$ & 149.817 & $\$$ & 190.732 & $\$$ & .915 & $-21,45 \%$ \\
\hline Arrendamiel & $\$$ & - & $\$$ & - & $\$$ & - & & $\$$ & 78.813 & $\$$ & & $\$$ & 78.813 & \\
\hline Contribución única temporal & $\$$ & & $\$$ & - & $\$$ & & & $\$$ & 7.764 & $\$$ & 14.865 & $\$$ & -7.101 & $-47,77 \%$ \\
\hline Impuestos diferidos & $\$$ & & $\$$ & - & $\Phi$ & - & & $\$$ & 464 & $\$$ & 18.205 & $\$$ & -17.741 & $-97,45 \%$ \\
\hline Total pasivos & $\$$ & 2.167 .544 & $\$$ & 2.077 .683 & $\Phi$ & 89.861 & $4,33 \%$ & $\$$ & 1.898 .748 & $\$$ & 2.427 .196 & $\$$ & -528.448 & $-21,77 \%$ \\
\hline Patrimonio & & & & & $\$$ & & & & & & & & & \\
\hline Capital social & $\$$ & 5.500 .000 & $\$$ & 5.500 .000 & $\$$ & - & $0,00 \%$ & $\$$ & 893.200 & $\$$ & 532.700 & $\$$ & 360.500 & $67,67 \%$ \\
\hline Aporte futura capitalización & $\$$ & & $\$$ & & $\$$ & - & & $\$$ & & $\$$ & & $\Phi$ & & \\
\hline Reservas & $\$$ & 209.170 & $\$$ & 209.170 & $\$$ & - & $0,00 \%$ & $\$$ & 32.114 & $\$$ & 32.114 & $\$$ & - & $0,00 \%$ \\
\hline Efectos NIFF'S & $\$$ & -133.999 & $\$$ & -133.999 & \$\$ & - & $0,00 \%$ & $\$$ & -14.398 & $\$$ & -14.398 & \$ & - & $0,00 \%$ \\
\hline Resultados acumulados & $\$$ & -86.347 & $\$$ & -81.547 & $\$$ & -4.800 & $5,89 \%$ & $\$$ & 135.562 & $\$$ & 39.937 & $\$$ & 95.625 & $239,44 \%$ \\
\hline Utilidades Reteni & $\$$ & & $\$$ & & $\$$ & & & $\$$ & 311.889 & $\$$ & 654.046 & $\$$ & -342.157 & $-52,31 \%$ \\
\hline Total patrimoni & $\$$ & 5.488 .824 & $\$$ & 5.493 .624 & $\$$ & -4.800 & $-0,09 \%$ & $\$$ & 1.358 .367 & $\$$ & 1.244 .399 & $\$$ & 113.968 & $9,16 \%$ \\
\hline Total pasivo y patrimonio & & 7.656 .368 & & 7.571 .307 & $\$$ & 85.061 & $1,12 \%$ & & 3.257 .115 & & 3.671 .595 & & -414.480 & $-11,29 \%$ \\
\hline
\end{tabular}

Fuente: Balances Generales de las Asociación 1 y 2 de los años 2020 y 2019. 
Tabla 13.

Efectos contables -Análisis Horizontal de Estados de Resultados.

\begin{tabular}{|c|c|c|c|c|c|c|c|c|c|c|c|c|c|}
\hline \multirow{2}{*}{ Cuentas Contables / Años } & \multicolumn{7}{|c|}{ Asociación 1} & \multicolumn{6}{|c|}{ Asociación 2} \\
\hline & & 2020 & & 2019 & & $\begin{array}{l}\text { Variación } \\
\text { Absoluta }\end{array}$ & $\begin{array}{l}\text { Variación } \\
\text { Relativa }\end{array}$ & & 2020 & & 2019 & $\begin{array}{l}\text { Variación } \\
\text { Absoluta }\end{array}$ & $\begin{array}{l}\text { Variación } \\
\text { Relativa }\end{array}$ \\
\hline \multicolumn{14}{|l|}{ Ingresos operacionales } \\
\hline Ventas netas & $\$$ & 2.082 .924 & $\$$ & 4.216 .673 & $\$$ & -2.133 .749 & $-50,60 \%$ & $\$$ & 2.877 .991 & $\$$ & 4.997 .381 & -2.119 .390 & $-42,41 \%$ \\
\hline Costo de ventas & $\$$ & 1.676 .845 & $\$$ & 3.608 .468 & $\$$ & -1.931 .623 & $-53,53 \%$ & $\$$ & 2.454 .153 & $\$$ & 3.666 .525 & -1.212 .372 & $-33,07 \%$ \\
\hline Utilidad bruta en ventas & $\$$ & 406.079 & $\$$ & 608.205 & $\$$ & -202.126 & $-33,23 \%$ & $\$$ & 423.838 & $\$$ & 1.330 .856 & -907.018 & $-68,15 \%$ \\
\hline \multicolumn{14}{|l|}{ Gastos operacionales } \\
\hline Gastos de administración & $\$$ & 138.584 & $\$$ & 439.527 & $\$$ & -300.943 & $-68,47 \%$ & $\$$ & 360.735 & $\$$ & 605.385 & -244.650 & $-40,41 \%$ \\
\hline Gastos de venta & $\$$ & 343.992 & $\$$ & 77.461 & $\$$ & 266.531 & $344,08 \%$ & $\$$ & 301.901 & $\$$ & 584.000 & -282.099 & $-48,30 \%$ \\
\hline Gastos operativos & $\$$ & 482.576 & $\$$ & 516.988 & $\$$ & $-34.412,00$ & $-6,66 \%$ & $\$$ & 662.636 & $\$$ & 1.189 .385 & $\$ \quad-526.749,00$ & $-44,29 \%$ \\
\hline \multicolumn{14}{|l|}{ Otros ingresos y gastos } \\
\hline Otros ingresos & $\$$ & 156.204 & $\$$ & 96.748 & $\$$ & 59.456 & $61,45 \%$ & $\$$ & 59.565 & $\$$ & 16.614 & 42.951 & $258,52 \%$ \\
\hline Gastos financieros & $\$$ & 77.447 & $\$$ & 145.562 & $\$$ & -68.115 & $-46,79 \%$ & $\$$ & 157.922 & $\$$ & 154.888 & 3.034 & $1,96 \%$ \\
\hline Otros resultados integrales & $\$$ & - & $\$$ & - & $\$$ & - & & $\$$ & 17.685 & $\$$ & 40.084 & -22.399 & $-55,88 \%$ \\
\hline Impuesto a la Renta & $\$$ & - & $\$$ & - & $\$$ & - & & & & $\$$ & 16.092 & 16.092 & $100,00 \%$ \\
\hline Ingresos por impuestos diferidos & $\$$ & - & $\$$ & - & $\$$ & - & & $\$$ & 72.938 & $\$$ & 13.873 & 59.065 & $425,76 \%$ \\
\hline Total otros ingresos y gastos & $\$$ & 78.757 & $\$$ & -48.814 & $\$$ & $29.943,00$ & $-61,34 \%$ & $\$$ & -7.734 & $\$$ & -100.409 & $92.675,00$ & $-92,30 \%$ \\
\hline $\begin{array}{l}\text { Resultado del ejercicio antes de } \\
\text { impuestos }\end{array}$ & $\$$ & 2.260 & $\$$ & 42.403 & $\$$ & $-40.143,00$ & $-94,67 \%$ & $\$$ & -246.532 & $\$$ & 41.062 & $\$ \quad-287.594,00$ & $-700,39 \%$ \\
\hline
\end{tabular}

Fuente: Estados de Resultados de las Asociaciones 1 y 2 de los años 2020 y 2019. 


\section{Análisis de los efectos contables en los estados financieros Balance general}

El total de los activos presenta un incremento del 1,12\% en la Asociación 1; y una reducción del 11,29\% en la Asociación 2 respecto del ejercicio 2019. Este resultado muestra un decrecimiento progresivo durante el año 2020.

En cuanto al activo corriente en el año 2020 existe una reducción del 18,81\% en la primera Asociación analizada, mientras que, en la segunda, la variación en menos es de $15,95 \%$. Complementando este análisis, se observa que la disponibilidad del efectivo disminuyó en la empresa 1 en 18,81\%, y en la empresa dos en 15,95\%. En el caso del inventario en las dos Asociaciones se determinó una reducción del $8 \%$ en promedio.

Con relación al activo no corriente, se evidencia un incremento del 15,68\% y 41,44\% en cada Asociación respectivamente.

Con relación al pasivo corriente existe un decremento del 41,79\% en la Asociación 1, la cuenta que presenta mayor reducción es otras cuentas por pagar (185\%); mientras que, la asociación 2 presenta un decremento del 23,19\% en su pasivo corriente con mayor impacto en la cuenta obligaciones con instituciones financieras $(-84,47 \%)$, lo que determina el cumplimiento con sus obligaciones de corto plazo.

En lo concerniente al pasivo corriente, la Asociación 1 muestra un incremento del 70,67\%, siendo la cuenta más representativa obligaciones con instituciones financieras (187,31\%). Es evidente que, en el transcurso del año 2020, la pandemia por COVID-19, incidió en la situación financiera de esta organización, lo que conllevó a que acceda a financiamiento de largo plazo para cubrir sus obligaciones de corto plazo.

El patrimonio de las dos Asociaciones no presenta una mayor variación respecto del año 2019, en el caso de la primera existe una contracción del 0,09\%, y en la segunda se evidencia un incremento del $9,16 \%$. 


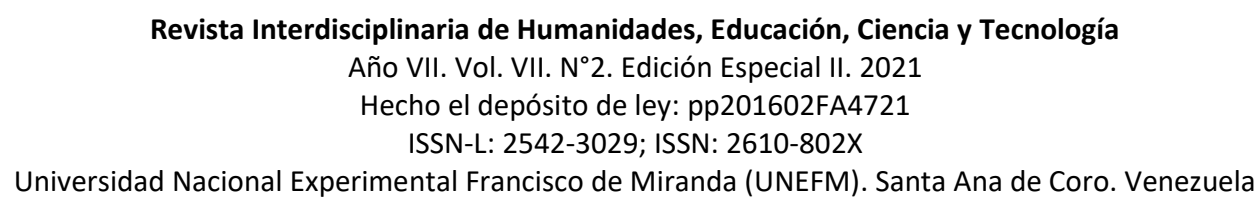

Gabriela Verónica Sevilla-Galarza; Lenyn Geovanny Vásconez-Acuña

\section{Estado de resultados}

El estado de resultados refleja el desempeño alcanzado en un periodo, esto puede ser utilidad o pérdida. En el caso de la Asociación 1, sus utilidades se redujeron en $94,67 \%$ con relación al año 2019, mientras que, la asociación dos presenta una pérdida notoria de su inversión en 700,39\%. De estos resultados se desprende la reducción de las ventas en un $50,60 \%$ y $42,41 \%$ respectivamente.

\section{Ratios}

La información obtenida de la Asociación 1 dedicada a la producción de textiles y confecciones refleja una imagen fiel de su patrimonio, situación financiera y resultados. En este sentido, el análisis de los indicadores de liquidez, endeudamiento, solvencia y rentabilidad que se presentan en la tabla 14 determinan su estructura y posición financiera en el periodo 2020.

Tabla 14. Efectos financieros.

\begin{tabular}{|c|c|c|}
\hline Ratios financieros & Razón & Interpretación \\
\hline $\begin{array}{l}\text { Liquidez } 2020=\frac{2^{\prime} 593.605}{713.063} \\
\text { Liquidez } 2019=\frac{3^{\prime} 194.599}{1^{\prime} 224.987}\end{array}$ & 3,64 & $\begin{array}{l}\text { En el año } 2020 \text {, el índice de liquidez de la asociación ascendió a } \\
3,64 \% \text {, quiere decir que la empresa contó con } \$ 3,64 \text { para } \\
\text { respaldar sus obligaciones por cada } \$ 1,00 \text { de deuda; a diferencia } \\
\text { del año } 2019 \text { que presentó } \$ 2,61 \text {. }\end{array}$ \\
\hline Endeudamiento $2020=\frac{2^{\prime} 167.544}{7^{\prime} 656.368}$ & 0,28 & $\begin{array}{l}\text { En el año } 2020 \text { el nivel de endeudamiento se encontró en } 0,28 \text {, } \\
\text { es decir que por cada } \$ 1,00 \text { que invirtió la empresa, } \$ 0,28\end{array}$ \\
\hline Endeudamiento $2019=\frac{2^{\prime} 077.683}{7^{\prime} 571.307}$ & 0,27 & $\begin{array}{l}\text { En tanto que, en el año } 2019 \text { el nivel de endeudamiento se } \\
\text { encontró en } \$ 0,27 \text {, esto significó que por cada } \$ 1,00 \text { que invirtió } \\
\text { la empresa, } \$ 0,27 \text { centavos fueron financiados por terceros. }\end{array}$ \\
\hline Solvencia $2020=\frac{7^{\prime} 656.368}{2^{\prime} 167.544}$ & 3,53 & $\begin{array}{l}\text { En el año } 2020 \text {, el índice de solvencia de la empresa fue de } \\
\$ 3,53 \text {, quiere decir que la organización mantuvo en su activo } \\
3,53 \text { USD por cada dólar que mantenía de deuda. }\end{array}$ \\
\hline Solvencia $2019=\frac{7^{\prime} 571.307}{2^{\prime} 077.683}$ & 3,64 & $\begin{array}{l}\text { En el año } 2019 \text {, el índice de solvencia de la empresa mostró que } \\
\text { disponía en sus activos de } \$ 3,64 \text {, por cada dólar adeudado. }\end{array}$ \\
\hline
\end{tabular}


Revista Interdisciplinaria de Humanidades, Educación, Ciencia y Tecnología

Año VII. Vol. VII. N². Edición Especial II. 2021

Hecho el depósito de ley: pp201602FA4721

ISSN-L: 2542-3029; ISSN: 2610-802X

Universidad Nacional Experimental Francisco de Miranda (UNEFM). Santa Ana de Coro. Venezuela

Gabriela Verónica Sevilla-Galarza; Lenyn Geovanny Vásconez-Acuña

$$
\begin{aligned}
& \text { ROA } 2020=\frac{2.260}{7^{\prime} 656,368} \times 100 \\
& \text { ROA } 2019=\frac{42.403}{7^{\prime} 571.307} \times 100
\end{aligned}
$$

0,03\% En el año 2020 el índice del ROA fue de 0,03\%, es decir que por cada dólar que se invirtió en los activos totales, se obtuvo $\$ 0,03$ centavos de utilidad neta. Mientras que, en el año 2019 el índice del ROA fue de $0,56 \%$, es decir que por cada dólar que se invirtió $0,56 \%$ en los activos totales se obtuvo $\$ 0,56$ centavos de utilidad neta.

Fuente: Estados financieros 2019 y 2020 de la Asociación 1. 


\section{Estrategias financieras para superar la crisis}

Las asociaciones para continuar con el negocio en marcha deben implementar estrategias financieras que les permitan mitigar los efectos de la pandemia, en la tabla 15 se presentan algunas alternativas que pueden ser consideradas.

\section{Tabla 15.}

\section{Estrategias financieras.}

\begin{tabular}{|c|c|c|c|}
\hline Tema & NIIF o NIC & Impacto & Estrategias \\
\hline Arrendamientos & NIIF 16 & $\begin{array}{l}\text { Incumplimiento de las obligaciones de pago de la renta } \\
\text { como arrendatario. } \\
\text { Retrasos en los pagos del canon de arrendamiento. }\end{array}$ & $\begin{array}{l}\text { Negociar y realizar concesiones, ampliaciones de plazos y reducción de costos. } \\
\text { Analizar la aplicación de deterioro de los derechos de uso. }\end{array}$ \\
\hline Inventarios & NIC 2 & Devaluación de inventarios & Reducir el valor en libros a menor costo o valor neto realizable. \\
\hline Propiedad, planta y equipo & NIC 16 & Subutilización de propiedad, planta y equipo & Realizar un estudio de la utilización y subutilización de las propiedades. \\
\hline $\begin{array}{l}\text { Amortizaciones de pérdidas } \\
\text { fiscales }\end{array}$ & NIC 12 & Pérdidas del ejercicio & $\begin{array}{l}\text { Cuantificar los costos y gastos extraordinarios generados por la pandemia. } \\
\text { Incorporar los costos y gastos a la cuenta costos indirectos. }\end{array}$ \\
\hline Contratos onerosos & NIC 37 & Costos superan los beneficios económicos & $\begin{array}{l}\text { Verificar las condiciones de los contratos. } \\
\text { Evaluar si los contratos pasan hacer contratos onerosos. }\end{array}$ \\
\hline Activos contingentes & NIC 37 & Cierre y suspensión de actividades económicas & Contratar seguros contra contingentes por cierre de actividades. \\
\hline Reconocimientos de ingresos & NIF 15 & Reducción de ventas & $\begin{array}{l}\text { Registrar las ventas cuando cancele el cliente, considerando concesiones, } \\
\text { devoluciones y multas. }\end{array}$ \\
\hline $\begin{array}{l}\text { Revelación de hechos } \\
\text { subsecuentes }\end{array}$ & NIC 10 & Afectación de balances financieros & $\begin{array}{l}\text { Reconocer y medir la afectación de activos y pasivos en los estados financieros } \\
\text { si estos ocurrieron posterior a la presentación de los informes. }\end{array}$ \\
\hline Negocios en marcha & NIC 1 & Incertidumbre de segur con el negocio en marcha & $\begin{array}{l}\text { Revelar en los estados financieros si el negocio tiene la capacidad de continuar } \\
\text { en marcha. } \\
\text { Emprender el despliegue de acciones para recuperar el efectivo y racionalizar } \\
\text { los costos de operación. }\end{array}$ \\
\hline Cartera de crédito & NIC 39 & Cartera vencida & $\begin{array}{l}\text { Incorporar condiciones de aplazamientos de pagos y mora. } \\
\text { Incorporar ajustes de garantías dinámicas que afecten a las estimaciones. }\end{array}$ \\
\hline
\end{tabular}


Revista Interdisciplinaria de Humanidades, Educación, Ciencia y Tecnología

Año VII. Vol. VII. N²2. Edición Especial II. 2021

Hecho el depósito de ley: pp201602FA4721

ISSN-L: 2542-3029; ISSN: 2610-802X

Universidad Nacional Experimental Francisco de Miranda (UNEFM). Santa Ana de Coro. Venezuela

Gabriela Verónica Sevilla-Galarza; Lenyn Geovanny Vásconez-Acuña

\begin{tabular}{llll}
\hline Cuentas por cobrar & NIIF 9 & Pérdida por remanente del activo & $\begin{array}{l}\text { Estimar la pérdida por remanente del activo por las condiciones actuales. } \\
\text { Aumentar estimaciones de deterioro como efecto de la operación. }\end{array}$ \\
\hline Beneficios para empleados & NIC 37 & Sobrestimaciones en los beneficios empleados & $\begin{array}{l}\text { Realizar un plan y determinar el valor presente de las obligaciones de los } \\
\text { trabajadores para tener un valor razonable y evitar sobrestimaciones. }\end{array}$ \\
\hline
\end{tabular}

Elaboración: Los autores. 


\section{CONCLUSIONES}

La crisis generada por la pandemia del Covid -19 ha desencadenado en una alta volatilidad e incertidumbre en los mercados, en este sentido, es importante que los negocios evalúen de manera permanente sus capacidades para hacer frente a las disrupciones operativas y financieras bajo el contexto de las medidas gubernamentales optadas en cada país.

Los mayores desafíos que están enfrentado las empresas ecuatorianas durante la pandemia, incluyen: reducción del flujo de efectivo debido a la disminución de las ventas y utilidades, trabajadores en cuarentena, disrupción de la cadena de suministro, inventarios perdidos, incremento de la cartera vencida, dificultades para cubrir las cuentas y documentos por pagar a corto y media plazo, entre otros. Bajo estas condiciones comerciales se evidencia una fuerte presión sobre el capital de trabajo y la liquidez de los negocios.

Los productores de textiles y confecciones de la ciudad de Pelileo concuerdan que las medidas tomadas por el gobierno ecuatoriano para enfrentar los efectos de la pandemia incidieron sobre la liquidez de sus empresas, además del aumento de los costos y gastos debido a la implementación de planes de bioseguridad para cuidar la salud de sus colaboradores.

Para medir el impacto contable por la pandemia se consideró como base los balances generales y los estados de resultados de dos asociaciones productoras de textiles y confecciones de los años 2019 y 2020, los principales resultados reflejan: disminución de las ventas, pérdidas económicas al finalizar el ejercicio 2020, reducción del disponible e incremento de los pasivos corrientes. Para gestionar la crisis se ha considerado pertinente identificar una serie de estrategias que los negocios pueden implementar para enfrentar los retos que supone la gestión del efectivo y a liquidez. 
Revista Interdisciplinaria de Humanidades, Educación, Ciencia y Tecnología

Año VII. Vol. VII. N². Edición Especial II. 2021

Hecho el depósito de ley: pp201602FA4721

ISSN-L: 2542-3029; ISSN: 2610-802X

Universidad Nacional Experimental Francisco de Miranda (UNEFM). Santa Ana de Coro. Venezuela

Gabriela Verónica Sevilla-Galarza; Lenyn Geovanny Vásconez-Acuña

\section{REFERENCIAS}

Apuntes Empresariales ESAN. (2020). Cómo gestionar el estado de flujo de efectivo en tiempos de pandemia [How to Manage Cash Flow Status in Pandemic Times]. Obtenido de https://n9.cl/2tfen

Arrarte-Mera, R. (2004). Importancia del Análisis Estratégico de los Estados Financieros Empresariales [Importance of Strategic Analysis of Business Financial Statements]. doi: https://doi.org/10.15381/quipu.v11i21.5485

Asociación para el progreso de la dirección (APD). (02 de 08 de 2019). ¿Cómo calcular el flujo de caja y para qué sirve? [How to calculate cash flow and what is it for?]. Obtenido de https://n9.cl/mltk3

Ballesteros-Cerchiaro, L. (16 de 02 de 2017). Análisis Financiero. Análisis vertical o estático [Vertical or static analysis], 3.2. Obtenido de https://n9.cl/w7f1p

Barreno, J. (18 de 01 de 2013). La importancia de medir la Rentabilidad [The importance of measure profitability]. Obtenido de https://n9.cl/jdhp8

Coronel-Morocho, J. (2019). Análisis del impacto financiero y tributario a partir de la valuación de inventarios. Revista Arbitrada Interdisciplinaria Koinonía, 4(2), 56-83. doi:http://dx.doi.org/10.35381/r.k.v4i2.467

Deloitte. (2020). COVID-19, Impactos en la Información Financiera [COVID-19, Impacts on Financial Information]. Obtenido de https://n9.cl/08ej9

Ernst \& Young EY. (2020). Consideraciones contables y financieras del Covid-19 [Covid19 accounting and financial considerations]. Obtenido de https://n9.cl/tkf7f

Espinoza-Carrión, D. (2019). La estrategia financiera como herramienta funcional para la gestión de la liquidez en las organizaciones. CIENCIAMATRIA, 5(1), 502-532. https://doi.org/10.35381/cm.v5i1.278

Fernández, R. (2020). Impacto económico y social del coronavirus (COVID-19) en España - Datos estadísticos [Economic and social impact of coronavirus (COVID19) in Spain - statistical data]. Statista, 1. Obtenido de https://cutt.ly/3/5EacT

Fondo Monetario Internacional. (2021). Enfrentar los peligros de un apalancamiento creciente [Addressing the dangers of increased leverage]. Obtenido de https://n9.cl/67yo2 
García-Jiménez, O. (2014). Fórmula DuPont y su rentabilidad, vista desde el punto administrativa [DuPont formula and its profitability, seen from the administrative point of view]. Inquietud Empresarial, 89-113.

González, O. (2021). Ratio financiero. conoce a profundidad el estado de tu empresa [Financial ratio. know in depth the state of your company]. Obtenido de aappvizer: https://n9.cl/abhaf

González-Lemus, A. (2020). Asociación Interamericana de Contabilidad AIC. Obtenido de El análisis financiero - La estrategia empresarial en tiempos de la pandemia Covid-19 [Financial analysis - The business strategy at the time of the Covid-19 pandemic]: https://n9.cl/o3vte

Guzmán-Maldonado, L. (2020). El estudio de flujos de efectivo, herramienta de análisis financiero ante el Covid-19- AIC [The study of cash flows, a financial analysis tool before the Covid-19- AIC]. Obtenido de Asociación Interamericana de Contabilidad: https://n9.cl/sxn93

Jaramillo, M. (2021). Liquidez de una empresa: Síntomas de cuando el efectivo está faltando [Company liquidity: Symptoms of when cash is missing]. Nubox. Chile. Obtenido de https://n9.cl/nr75b

Lozano-Meade, G. (2020). ¿Cómo optimizar su estrategia comercial y de precios en tiempo de crisis? [¿How to optimize your trading and pricing strategy in times of crisis?]. Obtenido de Building a better working world: https://n9.cl/idn03

Master MBA Sevilla. (2020). ¿Cuáles son los ratios de endeudamiento y cómo se calculan? [What are the debt ratios and how are they calculated?]. Obtenido de https://n9.cl/67yo2

Mejía-Hidalgo, P. (2020). Cómo analizar los indicadores financieros claves de las empresas en tiempos de Covid-19 [How to analyze key financial indicators of companies at Covid-19 times]. Gan@Más. Obtenido de https://n9.cl/p8zkj

Moncayo, C. (2016).¿Qué son actividades de inversión, operación y financiación en el estado de flujos? Obtenido de https://n9.cl/ov2a1

Mosquera-Choco, E. (2019). Estrategias Administrativas y Financieras: herramientas clave para una gestión. Revista Arbitrada Interdisciplinaria Koinonía, 4(1), 32-67. doi:http://dx.doi.org/10.35381/r.k.v4i1.371 
Nava-Rosillón, M. (2009). Análisis financiero: una herramienta clave para una gestión financiera eficiente [Financial analysis: a key tool for efficient financial management]. Obtenido de https://n9.cl/txirt

Navarro, P. (2019). Ratios financieros que deberías conocer [Financial ratios you should know]. Obtenido de https://contabilidadgamma.com/ratios-financieros/

Padilla-Martínez, M., Quispe-Otacama, A., \& Telencha-Santos, P. (2017). Aplicación de análisis financiera [Financial nalysis application]. Ambato: Centro de Contabilidad y Auditoría.

PricewaterhouseCoopers PWC. (2020). Obtenido de Implicaciones contables de los efectos del Covid-19 [Accounting implications of the effects of Covid-19]: https://n9.cl/01h9

Red de Instituciones Financieras de Desarrollo [Network of Financial Development Institutions]. (2020). Covid-19 y sus implicaciones en el Sistema Financiero Nacional. (Equifax, Ed.) Equifax, 6-9. Obtenido de https://n9.cl/99m0

Ricra-Milla, M. (2014). Análisis Financiero en las empresas [Financial analysis in companies]. Perú, Perú: Pacífico Editores.

Robles-Román, C. (2012). Fundamentos de administración financiera [Financial Analysis Application]. México: Red tercer Milenio S. C.

Sanabria, J. (2020). ¿Cuál es el impacto del COVID-19 en los estados financieros intermedios? [¿What is the impact of COVID-19 on interim financial statements?]. Obtenido de KPMG: https://n9.cl/yufs6

Touza, S., \& Picatoste, F. (2020). El impacto del COVID-19 sobre la liquidez y la solvencia de las empresas [The impact of COVID-19 on the liquidity and solvency of companies ]. Obtenido de Deloitte España: https://n9.cl/2tfcn

Alcázar-Ponce, J. (2015). Análisis fórmula Dupont ROA / ROE Financiero [Dupont ROA / ROE Financial Formula Analysis]. Obtenido de Linkeden: https://n9.cl/48myw 
Revista Interdisciplinaria de Humanidades, Educación, Ciencia y Tecnología

Año VII. Vol. VII. N². Edición Especial II. 2021

Hecho el depósito de ley: pp201602FA4721

ISSN-L: 2542-3029; ISSN: 2610-802X

Universidad Nacional Experimental Francisco de Miranda (UNEFM). Santa Ana de Coro. Venezuela

Gabriela Verónica Sevilla-Galarza; Lenyn Geovanny Vásconez-Acuña

@2021 por los autores. Este artículo es de acceso abierto y distribuido según los términos y condiciones de la licencia Creative Commons Atribución-NoComercial-Compartirlgual 4.0 Internacional (CC BY-NC-SA 4.0)

(https://creativecommons.org/licenses/by-nc-sa/4.0/ 